\title{
THE SPATIAL STRUCTURE OF TURBULENCE AT PRODUCTION WAVENUMBERS USING ORTHONORMAL WAVELETS
}

\author{
GABRIEL G. KATUL ${ }^{1,2}$ and MARC B. PARLANGE ${ }^{1,3}$ \\ ${ }^{1}$ Hydrologic Science, University of California, Davis, CA 95616; ${ }^{2}$ School of the Environment, Duke \\ University, Durham, NC 27708-0328; ${ }^{3}$ Department of Biological and Agricultural Engineering, \\ University of California, Davis, CA 95616
}

(Received in final form 19 December, 1994)

\begin{abstract}
Orthonormal wavelet expansions are applied to surface-layer measurements of vertical wind speed under various atmospheric stability conditions. The orthonormal wavelet transform allows for the unfolding of these measurements into space and scale simultaneously to reveal the large intermittent behavior in space for the turbulent production wavenumbers. Both Fourier and wavelet power spectra indicated the existence of a -1 power law for the vertical velocity measurements at the production wavenumbers. The -1 power law in the turbulent production range was derived from surface-layer similarity theory. A dimensionless skewness structure function is applied to the wavelet decomposed vertical velocity field to trace the destruction of the shear- or buoyancy-induced anisotropy under various stability conditions. The structure skewness function revealed shear- or buoyancy-induced eddy asymmetry dependence on stability at each scale within the -1 power-law wavenumber range with more isotropy during propagation from smaller to larger wavenumbers. The asymmetry of these events at the turbulent production wavenumbers appeared very localized in space, as well as in scale, and could be described with a simple eddy-overturning model. It is demonstrated that the wavelet transform is suitable for such analysis.
\end{abstract}

\section{Introduction}

It is well recognized that the statistical characteristics of atmospheric surface-layer (ASL) turbulent flow can be assumed to depend on the following dimensional parameters: 1) the friction velocity $\left.u_{*}=\left(\tau \rho^{-1}\right)^{1 / 2}, 2\right)$ the buoyancy parameter $\beta=g \gamma$, and 3) the sensible heat flux $\left(H=\rho c_{p}\left\langle w^{\prime} T^{\prime}\right\rangle\right)$ and the latent heat flux $\left(L_{v} E=\rho L_{v}\left\langle w^{\prime} q^{\prime}\right\rangle\right)$, where $\tau$ is the surface shear stress $=\rho\left\langle w^{\prime} u^{\prime}\right\rangle, w^{\prime}$ and $u^{\prime}$ are the fluctuations of the vertical and horizontal velocity, respectively, about the mean values, $\langle$.$\rangle is the averaging operator, \rho$ is the air density, $g$ is the gravitational acceleration, $\gamma=T^{-1}$ is the coefficient of thermal expansion (assuming air is an ideal gas), $T$ is the mean absolute temperature of the surface layer, $c_{p}$ and $L_{v}$ are the heat capacity and the latent heat of vaporization, respectively, and $q^{\prime}$ and $T^{\prime}$ are the specific humidity and temperature fluctuations around the mean values, respectively (e.g., Monin and Obukhov, 1954; Monin and Yaglom, 1971, Ch. 4; Brutsaert, 1982, Ch. 3, 4).

A recent study by Kader and Yaglom (1991) based on the work of Zilitinkevich (1971), Betchov and Yaglom (1971), and Kader and Yaglom (1984) suggests that the ASL is comprised of three sublayers that have a self preserving turbulence structure. These sublayers are 1) forced convective or dynamic sublayer, 2) dynamic convective, and 3) free convective. 
The forced convective sublayer occurs when $z \ll|L|$, where $z$ is the height above the ground surface and $L$ is the Obukhov length defined by

$$
L=-\frac{\rho u_{*}^{3}}{k g\left[\left(\frac{H}{T c_{p}}\right)+0.61 E\right]},
$$

where $k=0.4$ is the von Karman constant. Within the forced convective sublayer, the buoyancy parameter $\beta$ can be excluded from the list of dimensional variables. The free convective sublayer occurs when $z \gg|L|$ with $u_{*}$ omitted from the list of dimensional variables. The dynamic convective sublayer occurs at moderate values of $\zeta=-z / L$ and all three dimensional variables are important. The distinction between these sublayers allows for separate universal formulation of the Fourier power spectrum and co-spectrum $\left(E_{i j}\right)$ of the velocity components $(i=1,2,3 ; j=$ $1,2,3)$ for wavenumbers $(K)$ that are dynamically important and much smaller than the Kolmogorov dissipation scales (Kader and Yaglom, 1991).

For the vertical velocity fluctuations $w^{\prime}(i=3, j=3)$, the Fourier power spectrum $E_{33}(K)$ within the various sublayers has a universal power-law behavior given by (Kader and Yaglom, 1991):

$$
\begin{aligned}
& E_{33}(K)=\begin{array}{ll}
C_{33}^{(1)} K^{-5 / 3} & \text { for } b_{33}^{(1)}<K<a_{33}^{(1)} \\
G_{33}^{(1)} K^{-1} & \text { for } d_{33}^{(1)}<K<b_{33}^{(1)}
\end{array} \quad \text { For } z \ll L, \\
& E_{33}(K)=\begin{array}{l}
C_{33}^{(2)} K^{-5 / 3} \text { for } b_{33}^{(2)}<K<a_{33}^{(2)} \\
G_{33}^{(2)} K^{-5 / 3}
\end{array} \text { for } d_{33}^{(2)}<K<b_{33}^{(2)}
\end{aligned} \quad \text { For } z \gg|L|,
$$

where $a_{33}$ is the wavenumber at which the inertial subrange terminates, $b_{33}$ is the wavenumber at which the inertial subrange commences, and $d_{33}$ is a wavenumber at which energy is injected. It is now accepted that the energy cascade from lower to higher wavenumbers is the most important dynamical process through which the memory of the energy injection at production scales is lost and the universality of flow structure, as described by Kolmogorov's theory (1941), results at higher wavenumbers (Yamada and Ohkitani, 1991a,b; Lesieur, 1987, pp. 96-100; Argoul et al., 1989; Kraichnan, 1991; Monin and Yaglom, 1975, Ch. 8). For the forced and dynamic convective sublayers, (2) and (4) indicate that the injection memory, characterized by the asymmetry in the production scales, disperses as the energy cascades to higher wavenumbers following a -1 power law spectral behavior. When the cascading energy extends to inertial subrange wavenumbers, the flow becomes locally isotropic and the eddy asymmetry due to turbulent production is lost (Monin and Yaglom, 1975; Ch. 8). 
As shown by Tennekes and Lumley (1972; p. 264) and Kader and Yaglom (1991), the occurrence of the -1 power law at the production scales can be derived from dimensional arguments by noting that the spectrum of velocity for the small wavenumber range has units of $\left(\mathrm{m} \mathrm{s}^{-1}\right)^{2} \mathrm{~m}$. Therefore, the spectrum of the vertical velocity $E_{33}(K)$ for large scales, based on Monin-Obukhov (1954) similarity theory with velocity and length dimensions scaled by $u_{*}$ and $z$, results in

$$
\frac{E_{33}(K)}{u_{*}^{2} z}=\chi(K z, z / L)
$$

where $\chi($.$) is a dimensionless universal function. For the case when z \ll|L|$ (i.e., $|z / L| \rightarrow 0$ ), the stability parameter is no longer important (near neutral conditions). Therefore, $\chi($.$) depends only on the product K z$. In addition, if we assume that large-scale disturbances are greater than $z$, and that their statistical regime will not alter for moderate changes in $z$, then the spectrum $E_{33}(K)$ is no longer dependent on $z$. Hence, if $K z$ is small enough, we can eliminate $z$ from (5) by requiring

$$
\frac{E_{33}(K)}{u_{\star}^{2}}=\frac{A_{33}}{K z},
$$

which yields $E_{33}(K)=A_{33} u_{\star}^{2} \mathrm{~K}^{-1}$, where $A_{33}$ is a universal constant (Kader and Yaglom, 1984, 1991). The occurrence of a power-law behavior at production wavenumbers has been observed in many studies. The -1 power law was originally derived by Tchen (1953) for the longitudinal velocity spectrum $E_{11}(K)$ within the boundary layer of a flat plate. However, Tchen (1953) concluded that the spectra $E_{22}(K)$ and $E_{33}(K)$ will not exhibit a -1 power law since no production occurs in these two directions (at least for neutral density stratification). Perry and Abell (1975) and Perry et al. (1986) experimentally demonstrated the occurrence of a $K^{-1}$ power law for the longitudinal velocity spectrum $E_{11}(K)$ in fully developed pipe-flow turbulence, but precluded its existence for $E_{33}$, while Koroktov (1976) observed an $E_{11}(K) \sim K^{-1}$ in channel flows. Antonia and Raupach (1993), using rough-wall boundary-layer wind tunnel measurements demonstrated the existence of a-1 power law for both $E_{11}$ and $E_{33}$. Recently, a spectral large-eddy simulation (LES) of decaying isotropic turbulence convecting a passive temperature admixture was performed by Metais (1991) and Metais and Lesieur (1992) to study the spectral properties of turbulence at small waveumbers. In Metais (1991) and Metais and Lesieur (1992) a -1 power law was computed at the production end of the temperature spectrum for nearly one decade, which agrees with temperature wavelet and Fourier spectral measurements performed by Katul and Parlange (1994) and Kader and Yaglom (1991) in the atmospheric surface layer. Other studies, summarized in Raupach et al. (1991), also report the occurrence of a -1 power law at production wavenumbers in rough-wall turbulent boundary layers under neutral conditions. However, Raupach et al. (1991) and Antonia and Raupach (1993) postulated that the -1 power law does not exist in the atmospheric surface layer due to buoyancy 
forces. This controversy regarding the existence of a -1 power law in the spectrum of the vertical velocity has been the main motivation for this study.

The objectives of this study are 1) to investigate the possible existence of a -1 power law in the vertical velocity power spectrum, and 2) to describe the spatial structure of the vertical velocity for the scales corresponding to the -1 power law. For that purpose, we make use of orthonormal wavelet decomposition proposed by Meyer (1989), Daubechies $(1988 ; 1992)$ and Mallat $(1989 a, b)$ to explicitly probe the spatial structure of turbulence within the -1 power law for the vertical velocity. It is demonstrated that the orthonormal wavelet transform, in conjunction with a simple eddy-overturning model, can be used to trace the evolution of the eddy injection asymmetry in space and scale as larger wavenumbers are approached. Spatial details regarding shear-dominated and buoyancy-dominated interaction within the -1 power law subrange are considered. The formation of shear-generated eddy overturning and buoyancy generated updraft-downdraft events are first identified from dimensionless statistical measures such as the structure skewness at various scales. These events are examined in the space/scale domain using $w^{\prime}$ time series measurements at $80 \mathrm{~cm}$ above a uniform bare soil surface.

\section{Wavelet Transforms}

Wavelet transforms are recent mathematical tools based on group theory of squareintegrable functions that allow the decomposition of functions, signals, fields, or operators into space and scale (Farge, 1992 a). Continuous wavelet transforms were introduced by Grossmann and Morlet $(1984,1985)$ and have been applied to turbulence by many investigators (see, e.g., Farge, 1992a,b; Farge et al., 1992; Everson et al., 1990; Argoul et al., 198y, Barcy et al., 1991; Liandrat and MoretBailly, 1990; Mahrt,1991). The continuous wavelet transform has proved to be an effective tool in studying singularities (Farge, 1992a; David, 1992), fractal structure of turbulence (Everson et al., 1990), or identifying coherent motion in turbulence measurements (Collineau and Brunet, 1993). However, the continuous transform may not be advantageous if the interest is in energy aspects of turbulence because the kernel functions are not mutually orthogonal and no immediate physical meaning can be associated with the expansion coefficients (Yamada and Ohkitani, 1990; 1991a,b). Moreover, the continuous wavelet transform forms an overcomplete basis that can bring ahout undesired formal relations between the wavelet coefficients themselves (Yamada and Ohkitani, 1991a,b; Meyer, 1989). In brief, orthonormal wavelet expansions are the discrete version of the continuous wavelet transforms, but they form a complete basis with mutually orthogonal kernel functions (Meneveau, 1991a). This allows a clear and conventional physical interpretation of the expansion coefficients from the energetic point of view when applied to turbulence measurements (e.g., Yamada and Ohkitani, 1990; 1991a,b; Meneveau, 1991a,b; Katul and Parlange, 1994). 


\subsection{CONTINUOUS WAVELET TRANSFORMS}

As shown by Grossmann et al. (1989), the continuous wavelet transform of a real square integrable signal $f(x)$ with respect to a real integrable analyzing wavelet $\psi(x)$ may be defined as

$$
W(b, a)=c_{g}^{-1 / 2} \frac{1}{\sqrt{a}} \int_{-\infty}^{+\infty} \psi\left(\frac{t-b}{a}\right) f(t) \mathrm{d} t,
$$

where $a$ is a scale dilation, $b$ is a position translation, $W(b, a)$ is the wavelet coefficient at position $b$ and scale $a$, and $C_{g}$ is a finite constant defined by

$$
C_{g}=\int_{-\infty}^{+\infty}|K|^{-1}\left|\psi^{*}(K)\right|^{2} \mathrm{~d} K
$$

where $K$ is the wavenumber and $\psi^{*}$ is the Fourier transtorm of $\psi(x)$ given by

$$
\psi^{*}(k)=\int_{-\infty}^{+\infty} \psi(y) e^{-i k y} \mathrm{~d} y
$$

If $\psi(x)$ is a suitable analyzing wavelet, it satisfies the following conditions (Farge, 1992a): 1) the admissibility condition which requires that

$$
\int_{-\infty}^{+\infty} \psi(y) \mathrm{d} y=0
$$

2) the similarity condition which requires the scale decomposition to be obtained by translation and dilation of one function, and 3) the invertibility condition which requires at least one reconstruction formula for recovering the original signal exactly from its wavelet coefficients. Hence, any square integrable function $f(x)$ can be recovered from the wavelet coefficients by the reconstruction formula

$$
f(x)=C_{g}^{-1 / 2} \int_{0}^{+\infty} \int_{-\infty}^{+\infty} a^{-1 / 2} \psi\left(\frac{x-b}{a}\right) W(a, b) \frac{\mathrm{d} b \mathrm{~d} a}{a^{2}} .
$$

A popular example of a continuous wavelet is the second derivative of a gaussian given by $\psi(x)=\left(x^{2}-1\right) \exp \left(-x^{2} / 2\right)$. The wavelet transform is commonly compared to a microscope with the optics constructed by $\psi$, the enlargement given by $a$, and the position given by $b$ (Liandrat and Moret-Bailly, 1990; Collineau and Brunet, 1993).

\subsection{ORTHONORMAL WAVELET EXPANSIONS}

Much of this material is presented in Katul and Parlange (1994); however, for completeness the main findings are given here. For actual turbulence measurements, discrete wavelet transforms are preferred since $f(x)$ is generally sampled 
at only discrete points $x_{j}$ depending on the resolution of the sensor and the sampling frequency (see Mallat, 1989a,b; Madych, 1992). Therefore, it is necessary to discretize the scale and space domain of (7). It should be mentioned that the discretization of (7) is not arbitrary since conservation of the amount of information in the signal (Daubechies, 1988) is required to form a complete and mutually orthogonal basis. As shown by Daubechies (1988; 1992, pp. 10) and Mallat (1989 a,b), using a logarithmic uniform spacing for the scale discretization with increasingly coarser spatial resolution at larger scales (i.e., the position index is dependent on the scale index), a complete orthogonal wavelet basis can be constructed. These basis functions are given by

$$
\psi_{[j]}^{(m)}(y)=a_{0}^{-m / 2} \psi\left(\frac{y-j b_{0} a_{0}^{m}}{a_{0}^{m}}\right),
$$

where $m$ and $j$ are variable scale and position indexes, respectively, $a_{0}$ is the base of the dilation and $b_{0}$ is the translation length in units of $a_{0}^{m}$. Note how the translations are dependent on the scale dilations. The simplest and most efficient case for practical computations is the dyadic arrangement resulting in $a_{0}=2$ and $b_{0}=1$ (Daubechies, 1992, pp. 10; Chui, 1992, pp. 4; Mallat, 1989a,b). Hence, all scales along octaves $2^{m}$ and translations along $2^{m} j$ contribute to the construction of $f\left(x_{j}\right)=f(j)$ using

$$
f(j)=\sum_{m=1}^{m=\infty} \sum_{i=-\infty}^{i=+\infty} W^{(m)}[i] g^{(m)}\left[i-2^{m} j\right]
$$

where $g^{(m)}[i]$ is the discrete version of the wavelet function $\psi(x)$ at scale $m$. Details regarding the discretization of $\psi(x)$ are discussed later. The discrete function $g^{(m)}[i]$ satisfies the discrete orthogonality condition

$$
\sum_{k=-\infty}^{k=+\infty} g^{(m)}\left[k-2^{m} j\right] g^{(n)}\left[k-2^{n} j\right]=\delta_{i j} \delta_{m i n},
$$

where $\delta_{i j}$ is the Kronecker delta function. The discrete wavelet coefficients at scale index $m$ and position index $i$ can be obtained by the convolution

$$
W^{(m)}[i]=\sum_{j=-\infty}^{j=+\infty} g^{(m)}\left[i-2^{m} j\right] f(j) .
$$

They satisfy the conservation of energy condition

$$
\sum_{j=-\infty}^{+\infty} f(j)^{2}=\sum_{m=1}^{m=+\infty} \sum_{i=-\infty}^{i=+\infty}\left(W^{(m)}[i]\right)^{2},
$$


which is similar to Parseval's identity in Fourier series (Chui, 1992, pp. 12). In the above derivation, it was assumed that $f(j)$ extends to $\infty$. In general, the number of observations defining $f(j)$ is finite and the summations in the above equations should not extend to infinite domains. If $N=2^{M}$ is the number of observations (i.e., $N$ is an integer power of 2), then the scale index $m$ can vary from 1 to $\mathrm{M}$ $=\log _{2}(N)$ and the position index at scale $m$ varies from 1 to $N 2^{-m}$. Note how an increase in scale results in a reduction of spatial resolution (e.g., at $m=1$, we have $N / 2$ coefficients, at $m=2$ we have $N / 4$ coefficients, at $m=M$ we have 1 coefficient). Also note that the above arrangement conserves the number of wavelet coefficients $(=N-1)$ required to decompose a signal or a discrete function defined over the $N$ points.

This dyadic arrangement is well suited for turbulence studies since the smallscale features of the turbulent flow, which change rapidly compared to the largescale features, are characterized by more wavelet coefficients (Meneveau, 1991a,b). The decomposition of $f(j)$ and the determination of the wavelet coefficients is generally carried out by an efficient pyramidal scheme known as multiresolution analysis.

\subsection{MULTIRESOLUTION ANALYSIS}

In this section, multiresolution analysis developed by Mallat (1989a,b) within the framework of Meyer's notion of orthogonal wavelet basis is briefly reviewed. This review is intended to illustrate how $g^{(m)}[i]$ can be obtained from $\psi(x)$ and how the computation of the wavelet coefficients is carried out.

In general, turbulence-measuring devices can only sample a continuous process $f(x)$ at finite resolution $\mathrm{d} y$ (assumed unity for normalization purposes in this section). Following Meneveau (1992a) it can be shown that there exists a set of orthonormal basis functions $\phi^{(0)}(x-i)$ that, by translation only, generate $f(x)$ using

$$
f(x)=\sum_{i=-\infty}^{i=+\infty} s^{(0)}[i] \phi^{(0)}(x-i) \mathrm{d} x
$$

where $s^{(m=0)}[i]$ is defined by the convolution

$$
s^{(m=0)}[i]=\int_{-\infty}^{+\infty} f(x) \phi(x-i) \mathrm{d} x .
$$

In practice, $\phi(x)$ is selected to have a fast decay away from the origin and to satisfy the orthonormality condition

$$
\int_{-\infty}^{+\infty} \phi^{(0)}(x-i) \phi^{(0)}(x-j) \mathrm{d} x=\delta_{i j},
$$


so that $s^{(m=0)}[i]$ represents discrete samples of $f(x)$ at unit resolution (i.e. for scale index $m=0$ ) on a mesh $i$ of unit size (in practice the mesh size is $\mathrm{d} y$ ). Note also that the samples $s^{(m=0)}[i]$ are collected in an orthogonal manner. Consider $f(x)$ at a coarser resolution $R_{m}=2^{m}$ denoted by $f^{(m)}(x)$. Analogous to $(17), f^{(m)}(x)$ can be computed from the convolution

$$
f^{(m)}(x)=\sum_{i=-\infty}^{i=+\infty} s^{(m)}[i] \phi^{(m)}\left(x-2^{m} i\right)
$$

where $s^{(m)}[i]$ is given by

$$
s^{(m)}[i]=\int_{-\infty}^{+\infty} f(x) \phi^{(m)}\left(x-2^{m} i\right) \mathrm{d} x
$$

and $\phi^{(m)}(x)$ is the dilated version of $\phi^{(0)}$ which is given by

$$
\phi^{(m)}(x)=2^{-m / 2} \phi^{(0)}\left(\frac{x}{2^{m}}\right) .
$$

Consider the basis function at scale $(m+1)$ which is completely embedded in scale $(m)$. Mallat $(1989 \mathrm{a}, \mathrm{b})$ demonstrated that the coarse-grained version $s^{(m+1)}[i]$ can be obtained from $s^{(m)}[j]$ using

$$
s^{(m+1)}[i]=\sum_{j=-\infty}^{j=+\infty} h[j-2 i] s^{(m)}[j],
$$

where $h[u]$ is defined by

$$
h[u]=2^{-1 / 2} \int_{-\infty}^{+\infty} \phi^{0} \frac{y}{2} \phi^{0}(y-u) \mathrm{d} y .
$$

Similarly, the wavelet coefficients at scale $(m+1)$ can be obtained from $s^{(m)}$ by

$$
W^{(m+1)}[i]=\sum_{j=-\infty}^{j=+\infty} g[j-2 i] s^{(m)}[j],
$$

where $g[u]$ is defined by

$$
g[u]=2^{-1 / 2} \int_{-\infty}^{+\infty} \psi^{0}\left(\frac{y}{2}\right) \phi^{0}(y-u) \mathrm{d} y
$$

which corresponds to the wavelet function $\psi(x)$ dilated by a factor of 2 and sampled by the smoothing function $\phi(x)$. The filters $h(u)$ and $g(u)$ are known as 


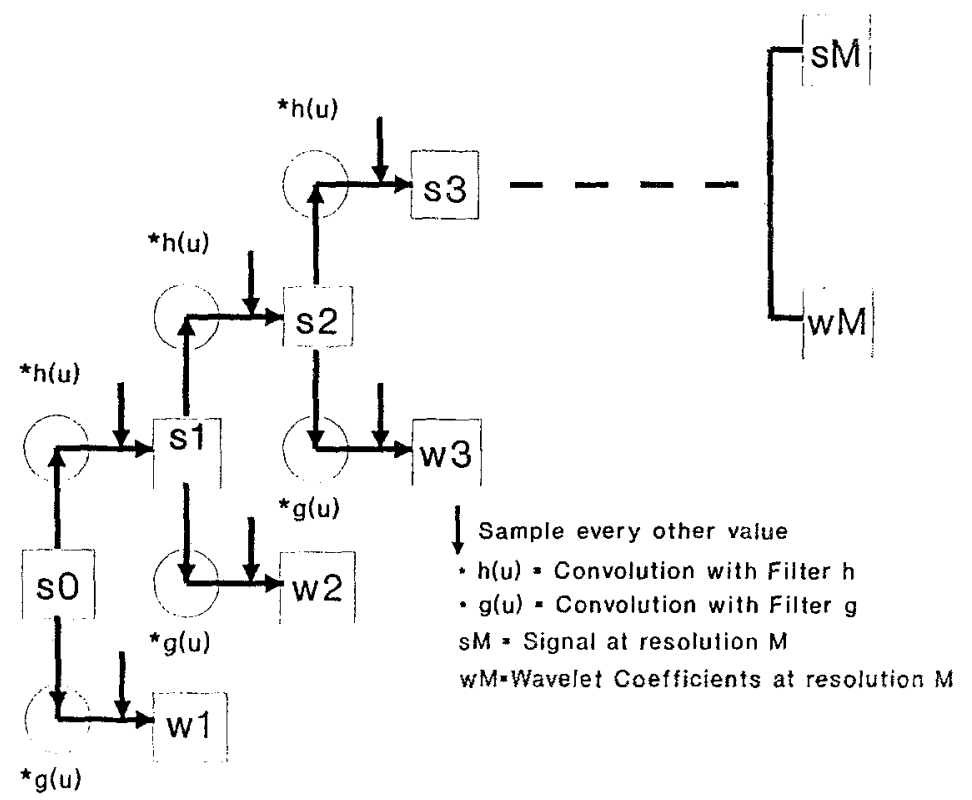

Fig. 1. Mallat's multiresolution pyramidal algorithm to obtain the wavelet coefficients as well as the coarse-grained signal at various scales from the Quadrature Mirror Filters $h(u)$ and $g(u)$.

the Quadrature Mirror Filters (QMF). Examples of QMFs for various compactly supported wavelets (i.e., wavelets that are zero everywhere except for a few points) are given in Daubechies (1992) and Katul and Parlange (1994). Equations (23) and (25) indicate that the coarse-grained version of $f(j)$ and the wavelet coefficients at a certain scale can be computed from the preceding finer scale. Figure 1 illustrates Mallat's (1989a,b) multiresolution algorithm for obtaining the wavelet coefficients and the coarse-grained series. We note in Figure 1 that $h(u)$ acts as a low pass filter which smooth the original series while $g(u)$ acts as a high pass filter that retains information lost in the smoothing. The algorithm stops when the signal reduces to a point. The algorithm presented in Figure 1 is general enough to allow implementation of various orthonormal wavelets such as the Daubechies wavelets (Daubechies, 1988) or the spline wavelets constructed by Battle-Meyer-Lemarie (see, e.g., Lemarie, 1988; Battle, 1987, 1992; Chui, 1992, pp. 177-214). However, for the case of the Haar wavelet (Haar, 1910), the scheme of Figure 1 reduces to a simple and efficient algorithm that does not require any convolution computations due to the compactness of the QMF of the Haar wavelet. Appendix A presents a fast wavelet algorithm (FWT) to compute the wavelet coefficients as well as the coarse-grained signal for the Haar wavelet. The Haar wavelet is adopted in this study since it has excellent localization in physical space (one vanishing moment) when compared to other wavelets. 


\subsection{PROPERTIES OF ORTHONORMAL WAVELETS}

Some properties of orthonormal wavelets are summarized below and more details are found in Meneveau (1991a).

1) All wavelet bases are orthonormal so that

$$
\int_{-\infty}^{+\infty} \psi^{(m)}\left(x-2^{m} i\right) \psi^{(n)}\left(x-2^{n} j\right) \mathrm{d} x=\delta_{i j} \delta_{m n} ;
$$

2) The wavelet bases are perpendicular to the smoothing or sampling function so that

$$
\int_{-\infty}^{+\infty} \phi^{(m)}\left(x-2^{m} i\right) \psi^{(m)}\left(x-2^{m} j\right) \mathrm{d} x=0
$$

3) The smoothing or sampling function is orthonormal to itself at any scale $m$ so that

$$
\int_{-\infty}^{+\infty} \phi^{(m)}\left(x-2^{m} i\right) \phi^{(m)}\left(x-2^{m} j\right) \mathrm{d} x=\delta_{i j} .
$$

Note that condition (2) suppresses undesired relations between the sampling function and the wavelet function.

\subsection{SPATIAl Statistics of TURbUlence}

From (16), the variance of the signal in terms of the wavelet coefficients can be computed from the conservation of energy

$$
\sigma^{2}=N^{-1} \sum_{m=1}^{m=M} \sum_{i=1}^{i=N}\left(W^{(m)}[i]\right)^{2},
$$

where $N$ is the number of observations, and $M=\log _{2}(N), m$ is the scale index $(1,2, \ldots, M)$, and $i$ is the position index. The total energy $T_{E}^{(m)}$ contained in scale $R_{m}=2^{m} \mathrm{~d} y$ is given by

$$
T_{E}^{(m)}=N^{-1} \sum_{i=1}^{i=2^{M-m}}\left(W^{(m)}[i]\right)^{2} .
$$

The wavenumber $K$ corresponding to scale $R_{m}$ is defined as $2 \pi\left(R_{m}\right)^{-1}$. Therefore, the wavelet power spectral density function (energy per wavenumber) at wavenumber $K$ is the ratio of $T_{E}^{(m)}$ to $\Delta K=\left(2 \pi 2^{-m} \mathrm{~d} y^{-1} \ln (2)\right)$ which, after some simplification, reduces to

$$
E(K)=\left\langle\left(W^{(m)}[i]\right)^{2}\right\rangle\left(\frac{\mathrm{d} y}{2 \pi \ln (2)}\right)
$$


where $\langle$.$\rangle is averaging in space over all values of i$ (not to be confused with time averaging). The spatial standard deviation about this power spectrum can also be computed from

$$
S D_{E}(K)=\left(\frac{\mathrm{d} y}{2 \pi \ln (2)}\right)\left[\left\langle W^{(m)}[i]^{4}\right\rangle-\left\langle\left(W^{(m)}[i]\right)^{2}\right]^{1 / 2} .\right.
$$

A plot of $E(K)$ and $E(K)+S D_{E}(K)$ gives a compact representation of the energy and its spatial variability at each scale. Such a representation is referred to as a "dual spectrum" (see Meneveau, 1991a,b). Also, other spatial statistical measures, such as the Flatness Factor $(F F)$, can be computed for wavenumber $K$ using

$$
F F(K)=\frac{\left\langle\left(W^{(m)}[i]\right)^{4}\right\rangle}{\left\langle\left(W^{(m)}[i]\right)^{2}\right\rangle^{2}}
$$

\section{Dimensionless Structure Skeweness}

Having introduced the wavelet transform as a tool for decomposing the turbulence measurements into space and scale, we focus on statistical measures that are employed to identify the nature of the eddy asymmetry and its propagation through the production range to higher wavenumbers. The asymmetry of the motion as well as the degree of anisotropy of turbulence at scale $R_{m}=2^{m} \mathrm{~d} y$, in relation to the mean shear, can be examined using the structure skewness $(S S)$ defined by

$$
S S^{(m)}(r)=\frac{\left[w^{(m)^{\prime}}(x)-w^{(m)^{\prime}}(x+r)\right]^{3}}{\left[\left(w^{(m)^{\prime}}(x+r)-w^{(m)^{\prime}}(x)\right)^{2}\right]^{3 / 2}},
$$

where $w^{(m)^{\prime}}(x)$ is the fluctuation of the vertical velocity (obtained from the multiresolution decomposition previously described) at position $x=\left(2^{m} \mathrm{~d} y j\right)$ and scale $2^{m} \mathrm{~d} y$. Here $\mathrm{d} y(=V \mathrm{~d} t)$ is determined from Taylor's (1938) hypothesis, where $V$ is the mean horizontal wind speed, and $\mathrm{d} t$ is the sampling time interval.

As shown by Mahrt and Gamage (1987), the magnitude of $S S$ can be interpreted with regard to the general anisotropy and intermittency of the flow at that scale. The sign of $S S$ can be interpreted as a result of two possible mechanisms (see Figure 2): 1) negative $S S$ resulting from localized shear overturning events at the present scale, and (2) positive structure skewness due to localized individual updraft-downdraft events active at the present scale (for further details, see Mahrt and Gamage, 1987).

\section{Experiments}

Experiments were carried out over a $500 \mathrm{~m} \times 500 \mathrm{~m}$ uniform bare soil surface with a momentum roughness height $z_{0}$ of $2 \mathrm{~mm}$ (Katul and Parlange, 1992; Parlange 


\section{IDEALIZED MODELS FOR STRUCTURE SKEWNESS}

1) Shear Overturning

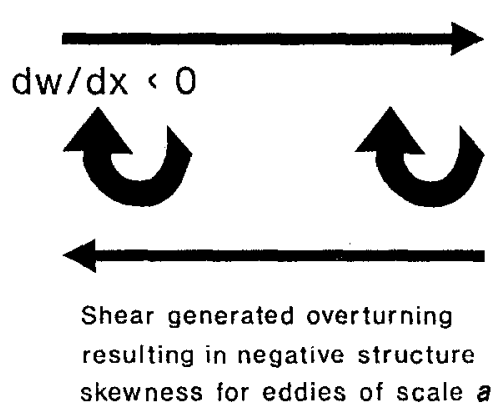

2) Drafts

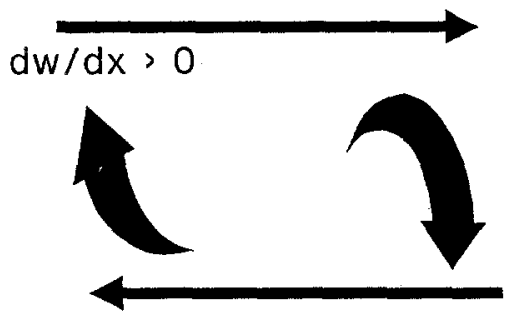

Updrafts and downdrafts contributing

to positive structure skewness for eddies of size a

Fig. 2. Two possible interpretations for the sign of the structure skewness. The first case is due to shear overturning resulting in a negative structure skewness. The second is due to updraft-downdraft events resulting in a positive structure skewness.

and Katul, 1992). A Campbell Scientific one-dimensional sonic anemometer (path length $=15 \mathrm{~cm}$ ) situated at $z=80 \mathrm{~cm}$ above the ground was used to monitor the fluctuations in the vertical velocity. The sampling frequency was set to $f_{s}=10 \mathrm{~Hz}$. Two 28-min experiments $(N=16,384)$ were carried out under unstable and stable atmospheric stability conditions as shown in Table I. Since this study is concerned with wavelet transforms as variance decomposition statistical tools, it is important to check the level of variance error due to the finite duration of the sampling period. As shown by Lumley and Panofsky (1964,pp. 37), for a given sampling period $T_{L}$ the approximate error $(\epsilon)$ in the variance measurement can be determined from

$$
\epsilon=\sqrt{2 \frac{\left\langle\left(w^{\prime}\right)^{2}\right\rangle}{\left\langle w^{2}\right\rangle} I_{t} \frac{1}{T_{L}}},
$$

where $I_{t}$ is the integral time scale determined by integrating the area under the autocorrelation function up to the first zero crossing (see Sirivat and Warhaft, 1983). Using the computed integral time scales reported in Table I, and using a sampling period of 28 minutes, the maximum error in the variance level is less than $4 \%$ for both experiments. Supporting measurements of temperature and humidity fluctuations using a fine wire chromel constant thermocouple $(0.0127 \mathrm{~mm})$ and a krypton hygrometer (path length $=1.105 \mathrm{~cm}$ ) were also carried out to obtain the sensible and latent heat fluxes, respectively. An estimate of $u_{*}$ was obtained from a Young triaxial light propeller anemometer situated at $3 \mathrm{~m}$ (distance constant $=1$ $\mathrm{m}$, sampling frequency $=1 \mathrm{~Hz})$. These measurements $\left(u_{*}, H\right.$, and $\left.L_{v} E\right)$ were used to estimate the Obukhov length during each experiment. Mean horizontal wind 


\section{TABLE I}

Meteorological conditions during the two experiments. The net radiation $\left(R_{n}\right)$ was measured by a Frischen type net radiometer, the soil heat flux $(G)$ was measured by a Thorntwaite soil heat flux plate, and the air temperature (at $80 \mathrm{~cm}$ ) was measured by a Campbell Scientific temperature probe. The time (PST) indicates the start of the 1-D sonic anemometer experiment. Also, the variance in $w^{\prime}\left(\operatorname{Var}\left(w^{\prime}\right)\right)$ and the computed integral time scales $\left(I_{T}\right)$ from the autocorrelation function for each experiment are tabulated. Taylor's hypothesis can be used to convert integral time scales to integral length scales

\begin{tabular}{lllllllllllll}
\hline $\begin{array}{l}\text { Day } \\
1992\end{array}$ & $\begin{array}{l}\text { Time } \\
\text { HHMM }\end{array}$ & $\begin{array}{l}\text { Run } \\
\text { No. }\end{array}$ & $\begin{array}{l}R_{n} \\
\mathrm{~W} \mathrm{~m}^{-2}\end{array}$ & $\begin{array}{l}G \\
\mathrm{~W} \mathrm{~m}^{-2}\end{array}$ & $\begin{array}{l}{ }^{\circ} \mathrm{C} \\
\mathrm{C}\end{array}$ & $\begin{array}{l}V \\
\mathrm{~m} \mathrm{~s}^{-1}\end{array}$ & $\begin{array}{l}u_{*} \\
\mathrm{~m} \mathrm{~s}^{-1}\end{array}$ & $\begin{array}{l}H \\
\mathrm{~W} \mathrm{~m}^{-2}\end{array}$ & $\begin{array}{l}L \\
\mathrm{~m}\end{array}$ & $\begin{array}{l}I_{T} \\
\mathrm{~s}\end{array}$ & $z / L$ & $\begin{array}{l}\operatorname{Var}\left(w^{\prime}\right) \\
\mathrm{m}^{2} \mathrm{~s}^{-2}\end{array}$ \\
\hline 280 & 1332 & 1 & 328.7 & 105.8 & 32.0 & 1.72 & 0.16 & 173.1 & -2.4 & 1.3 & -0.33 & 0.044 \\
285 & 2139 & 2 & -85.5 & -40.3 & 16.6 & 1.71 & 0.064 & -4.76 & -5.1 & 0.5 & 0.16 & 0.014 \\
\hline
\end{tabular}

speed was measured at $80 \mathrm{~cm}$ by a 3 -cup photochopper anemometer (sensitivity $=$ $\pm 0.2 \mathrm{~m} \mathrm{~s}^{-1}$ ). The turbulent intensity at $80 \mathrm{~cm}$ for both experiments was $<0.5$ and Taylor's (1938) frozen turbulence hypothesis was used (see Lumley, 1965; Powell and Elderkin, 1974; Stull, 1988, pp. 6).

\section{Results and Discussion}

The vertical velocity fluctuations as a function of time are shown for unstable (Figure 3a) and stable (Figure $3 b$ ) atmospheric stability conditions. It is apparent from Figures $3 a$ and $3 b$ that the magnitude of the vertical velocity fluctuations are much larger for unstable than for stable conditions. No apparent trend in the mean vertical velocity was observed for either of the 28 -min experiments.

\subsection{FOURIER AND WAVELET SPECTRA}

The Fourier power spectra for $N=16384$ observations (per experiment) were computed by dividing the signal into 32 sections, cosine tapering $5 \%$ on each side, and averaging the power (energy per unit wavenumber) at each wavenumber (see Shumway, 1988; Press et al., 1992). The Fourier power spectra for unstable and stable atmospheric stability conditions are shown in Figures $4 a$ and $4 b$, respectively. The existence of a power law consistent with a -1 exponent for both stability conditions is observed for at least 1 decade (see Figures $4 a$ and $4 b$ ). The regression model $\log (E)=\mathrm{A} \log (K)+B$ was used to determine the best fit line through the measurements presented in Figures $4 \mathrm{a}$ and $4 \mathrm{~b}$. The results are shown in Table II. The $-5 / 3$ power law commonly noted in atmospheric measurements (e.g., Kaimal et al., 1972) was not observed in the Fourier spectra presented in Figures 4a and $4 \mathrm{~b}$ for the $10 \mathrm{~Hz}$ sampling frequency. To further investigate the absence of the 


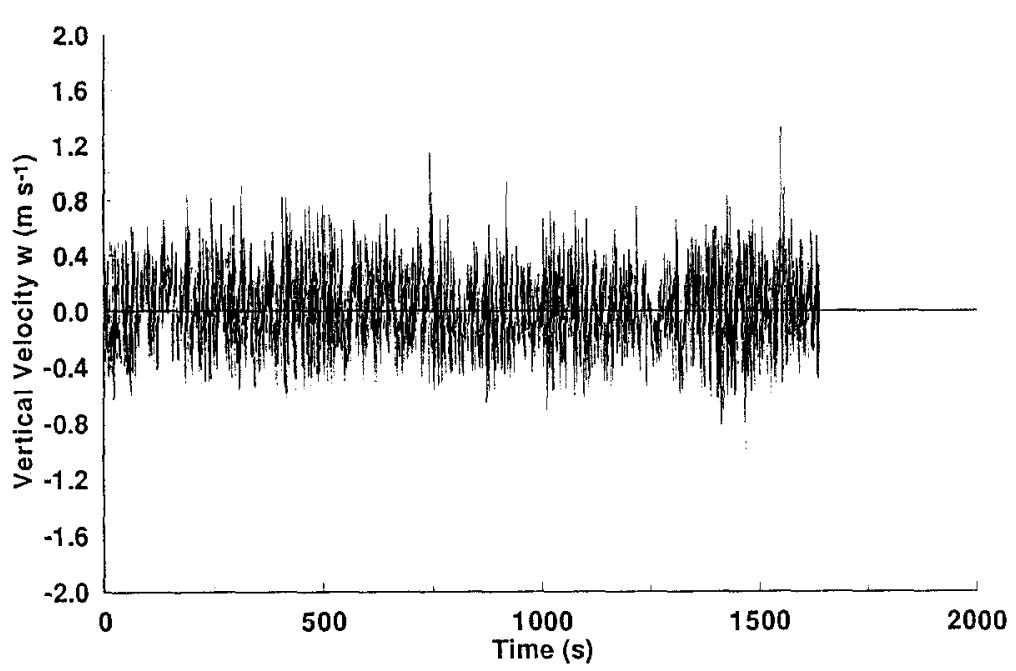

Fig. 3a. Measurements of the vertical velocity fluctuations for experiment 1 at $z=80 \mathrm{~cm}(N=$ 16384) for unstable atmospheric conditions.

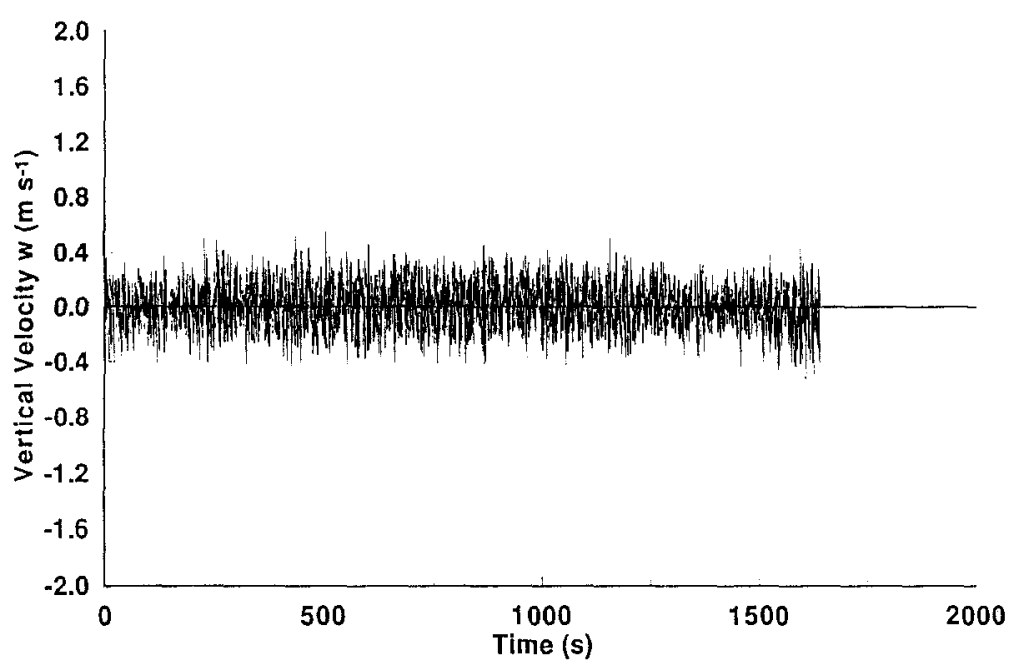

Fig. 3b. Same as Figure 3 a but for experiment 2 and stable atmospheric conditions.

$-5 / 3$ power law, we determined the maximum normalized frequency $\left(f_{n}\right)$ using $f_{s} z /\langle U\rangle(=10 \times 0.8 / 1.7=4.8)$. The vertical velocity spectra $\left(E_{33}\right)$ of Kaimal et al. (1972) show a clear inertial subrange for $f_{n}>5$, which is larger than the maximum $f_{n}$ in this study. Hence, the absence of a $-5 / 3$ power law appears to be due to 1) low sampling frequency and 2) the closeness of the measurement height to the ground surface. Several other wavenumber criteria regarding the commencement of the inertial subrange have been proposed. As shown by Monin and Yaglom (1975, pp. 457), a true inertial subrange exists when $n_{l} z /\langle U\rangle \sim 10$, where $n_{l}$ is the minimum 


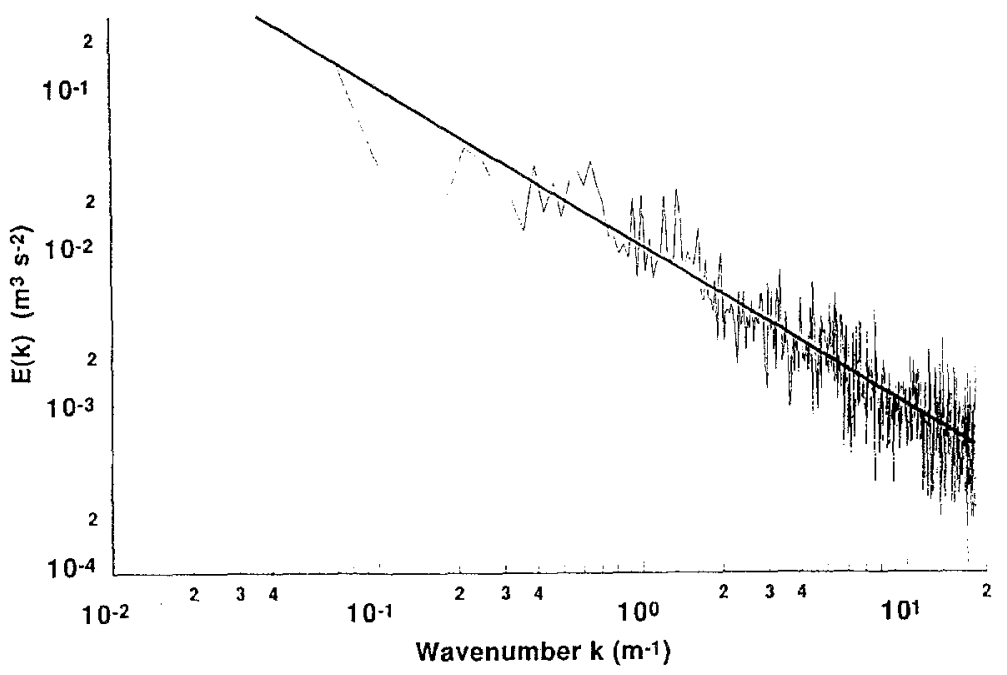

Fig. 4a. Fourier power spectrum of the vertical velocity fluctuations for experiment 1 (unstable conditions). Taylor's hypothesis was used to convert the time domain to the wavenumber domain. The -1 power law is also shown.

frequency necessary to capture the lower limit of the inertial subrange. However, Kaimal et al. (1972) showed that an inertial subrange can exist when $n_{l} z /\langle U\rangle \sim 5$ for unstable conditions and 10 for stable conditions. For both experiments, if we set $n_{l}=f_{s}$ (i.e., the frequency corresponding to the smallest resolvable scales by the instrument), then $f_{s} z /\langle U\rangle \sim 4.8$ which is less than 10. It appears that the Kaimal et al. (1972) criterion appears to underpredict the wavenumber at which the inertial subrange exists. We note that using $X$-probe measurements, Busch (1973) also showed that the Kaimal et al. (1972) inertial subrange wavenumber limits are rather small.

The two vertical velocity signals collected are decomposed into space and scale using the Multiresolution algorithm presented in Appendix A. This resulted in $\log _{2}(16384)=14$ octaves (i.e., the scale domain is discretized by 14 points) for each experiment. The Haar wavelet spectra for both stability conditions are shown in Figure 5a. Notice from Figure 5a that the area under the wavelet spectrum for the unstable case is much larger than for the stable case. Similar to the Fourier power spectra, a -1 power law relationship was observed in the Haar wavelet power spectra for one decade (see Figure 5a and Table II). Notice that the wavelet spectra are computed over a wider wavenumber range (compare the abscissa of Figures $4 \mathrm{a}$ and 5a) than the Fourier power spectra. Generally, to obtain a reliable estimate of the Fourier power spectrum, windowing and tapering are necessary. In contrast, windowing should be avoided when computing wavelet spectra since the spatial location of events is critical to the decomposition. Generally, windowing or averaging distorts the space-scale relation between the wavelet coefficients. 


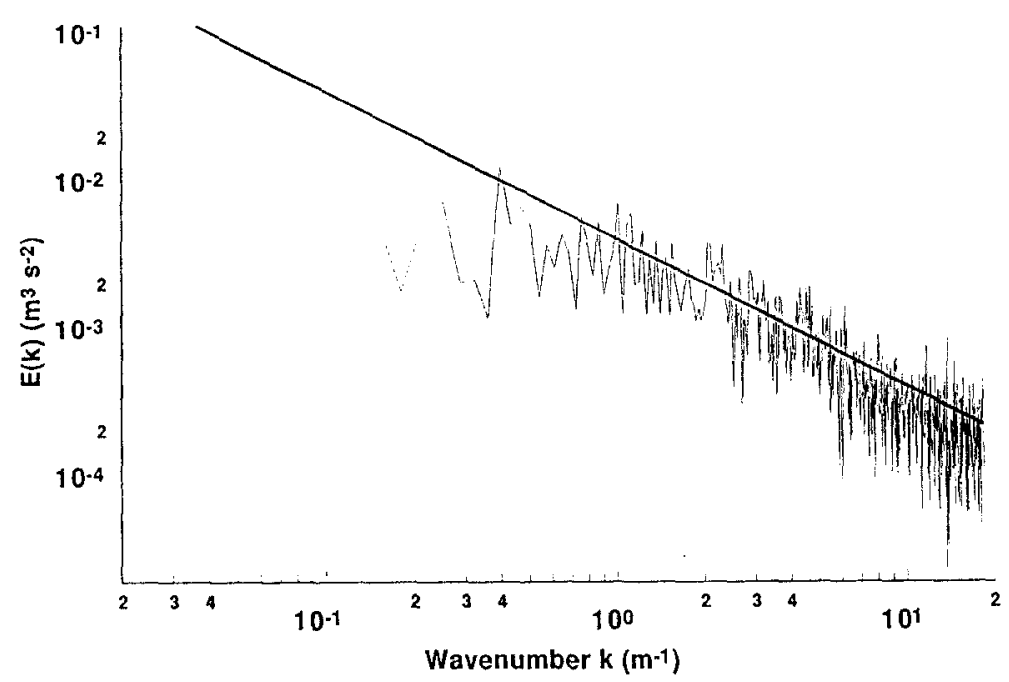

Fig. 4b. Same as Figure 4a but for experiment 2 (stable conditions).

TABLE II

The existence of a -1 power law. Regression analysis on the model $\log [E(K)]=A \log [K]+B$ for both Fourier and Wavelet Spectra is presented. The coefficient of correlation $\left(r^{2}\right)$, the standard error of estimate (SEE) as well as the wavenumber range $\left(K_{\min }-K_{\max }\right)$ used in the regression analysis are displayed for both experiments

\begin{tabular}{lllllllll}
\hline $\begin{array}{l}\text { Day } \\
1992\end{array}$ & $\begin{array}{l}\text { Time } \\
\text { HHMM }\end{array}$ & $\begin{array}{l}\text { Run } \\
\text { No. }\end{array}$ & $\begin{array}{l}\text { Minimum } \\
\text { Wavenumber } \\
\left(\mathrm{m}^{-1}\right)\end{array}$ & $\begin{array}{l}\text { Maximum } \\
\text { Wavenumber } \\
\left(\mathrm{m}^{-1}\right)\end{array}$ & $\begin{array}{l}\text { Slope } \\
(\mathrm{A})\end{array}$ & $\begin{array}{l}\text { Intercept } \\
(\mathrm{B})\end{array}$ & $r^{2}$ & SEE \\
\hline $\begin{array}{l}\text { Fourier Spectra } \\
280\end{array}$ & 1332 & 1 & 4.62 & 18.48 & -0.994 & -2.06 & 0.32 & 0.22 \\
285 & 2139 & 2 & 4.62 & 18.48 & -1.02 & -2.55 & 0.30 & 0.24 \\
Wavelet Spectra & & & & & & & \\
280 & 1332 & 1 & 2.309 & 18.48 & -0.98 & -1.99 & 0.998 & 0.019 \\
285 & 2139 & 2 & 2.309 & 18.48 & -0.99 & -2.42 & 0.999 & 0.032 \\
\hline
\end{tabular}

\subsection{SPATIAL STATISTICS OF THE POWER AT PRODUCTION WAVENUMBERS}

In studying the spatial variation of the energy in space, we depart from Meneveau's $(1991, a)$ dual-spectral representation. Instead of presenting the dual spectra as 


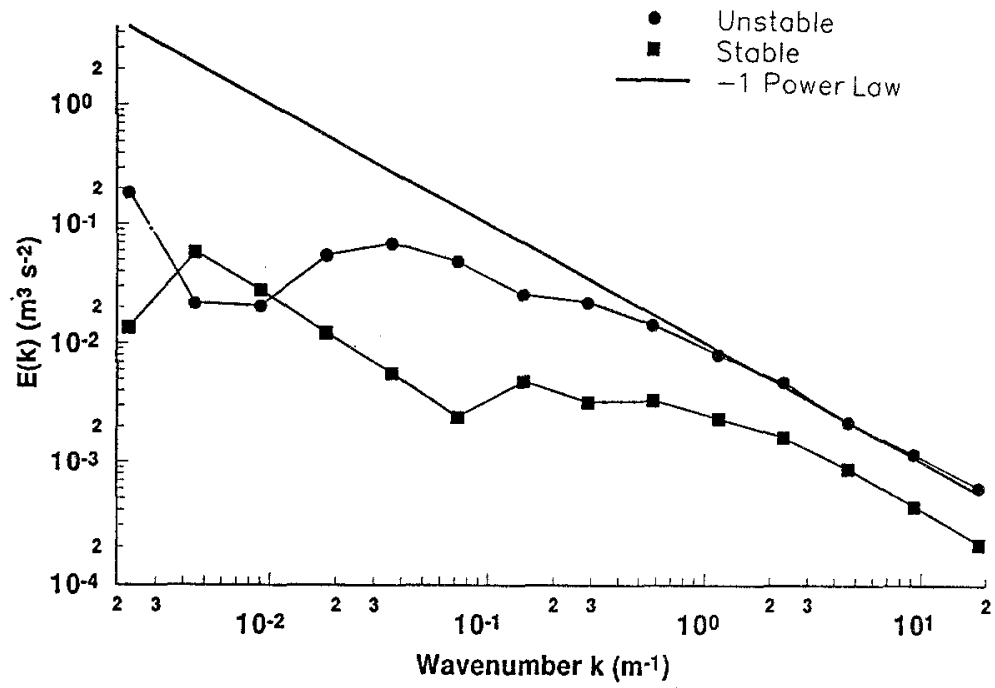

Fig. 5a. The Haar wavelet power spectra $E(K)$ for both experiments. Taylor's hypothesis was used to convert the time domain to the wavenumber domain. The -1 power law is also shown.

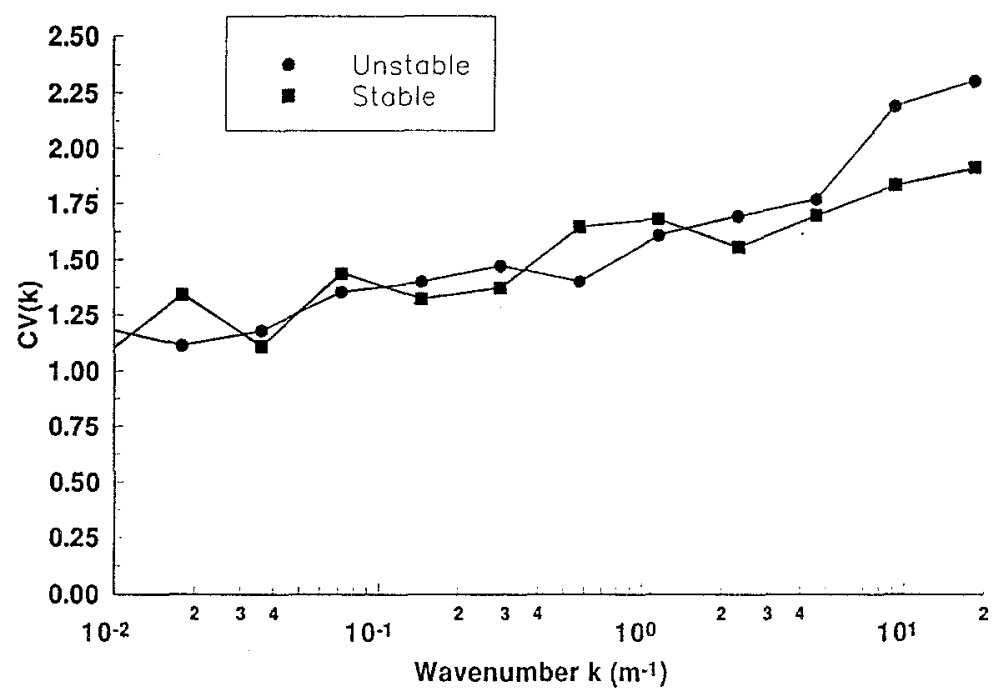

Fig. 5b. The coefficient of variation $C V(k)=S D_{E}(K) / E(K)$ as a function of wavenumber $(K)$ for both experiments.

$E(k)+S D E(K)$, we define a dimensionless coefficient of variation $C V(K)$ given by

$$
C V(K)=\frac{S D_{E}(K)}{E(K)}=\frac{\left[\left\langle W^{(m)}[i]^{4}\right\rangle-\left\langle\left(W^{(m)}[i]\right)^{2}\right\rangle^{2}\right]^{1 / 2}}{\left\langle\left(W^{(m)}[i]\right)^{2}\right\rangle}
$$




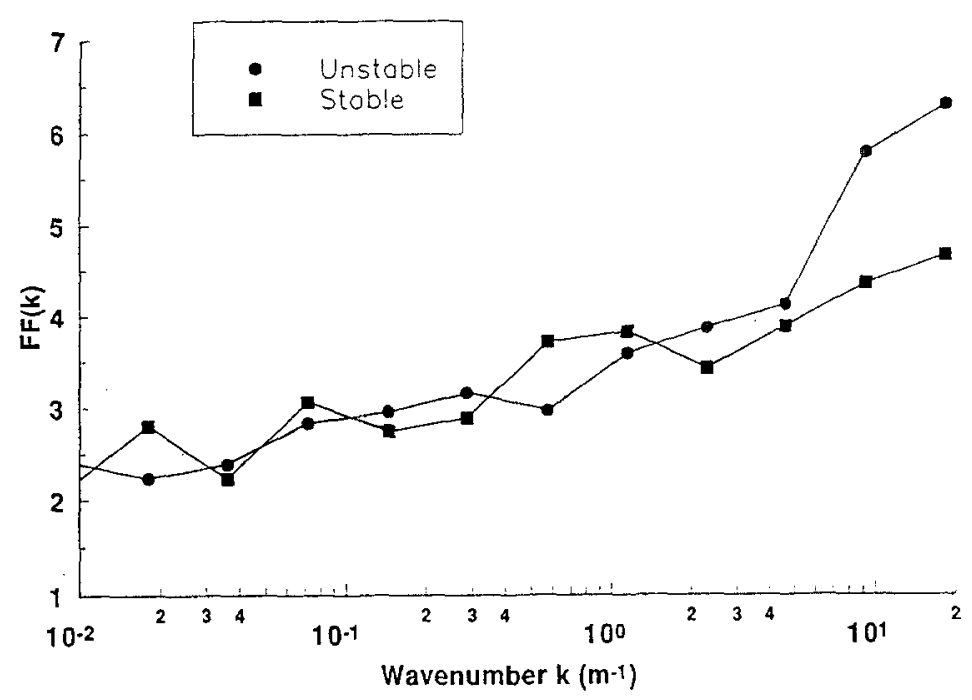

Fig. 5c. The wavelet flatness factor $(F F)$ as a function of wavenumber $(K)$ for both experiments.

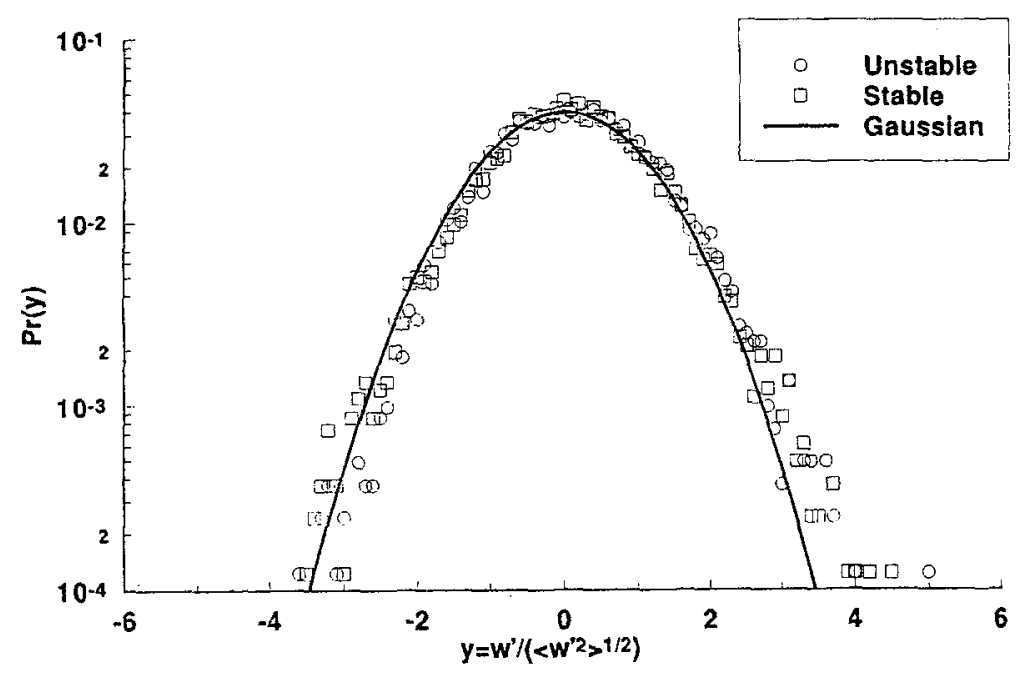

Fig. 5d. The probability density function $\operatorname{Pr}(y)$ of the vertical velocity fluctuations normalized by the vertical root-mean square velocity $(y)$ for both experiments. The solid line is the probability density function of a zero-mean and unit variance Gaussian process. The ordinate axis is logarithmic to emphasize the tails of $\operatorname{Pr}(y)$.

A plot of $C V(K)$ as a function of $K$ displays the relative spatial dispersion of the power around its mean value at wavenumber $K$. Recall that Fourier power spectra assume that the power is uniformly distributed in space with $C V(K)=0$ for all $K$. Figure $5 \mathrm{~b}$ shows the variation of $C V(K)$ for both stability conditions. Notice that for both cases, $C V(K)>1$, indicating that the spatial variation of the power 
$\left(S D_{E}(K)\right)$ is larger than its mean value $E(K)$. Also, it is evident from Figure $5 \mathrm{~b}$ that $C V(K)$ increases with increasing $K$ indicating increased spatial fluctuation in the power at smaller scales. In order to check whether stability influences the spatial fluctuation of the power at production wavenumbers $\left(K \sim 2 \mathrm{~m}^{-1}\right.$ to 18 $\mathrm{m}^{-1}$ ), we compare the magnitudes of $C V(K)$ for the two signals in Figure $5 \mathrm{~b}$. This comparison may indicate that spatial fluctuation in the power is larger for the unstable than for the stable case.

\subsection{SPATIAL STATISTICS OF THE GRADIENT AT FRODUCTION WAVENUMBERS}

The statistical properties of the vertical velocity gradient at wavenumber $K$ can be investigated using the wavelet flatness factor given by (34). Recall that the Haar wavelet transform amplifies the horizontal differences in the signal (see Appendix B). Hence, the dimensionless wavelet flatness factor is the same for differences or gradients since the division by the scale (a) required to convert differences to gradients in (34) cancels out. The variation of the wavelet flatness factor as a function of $K$ is presented in Figure $5 c$ for both stability conditions. Notice in Figure $5 \mathrm{c}$ that the wavelet flatness factor is nearly 3 (i.e. nearly Gaussian) for wavenumbers smaller than the production wavenumbers. Also, from Figure $5 \mathrm{c}$ notice that the wavelet flatness factor increases with increasing wavenumber for both stability conditions. This indicates that the gradients (as approximated by the wavelet transformed coefficients) tend to be non-Gaussian at the smaller scales (or larger wavenumbers), although the vertical velocity is nearly Gaussian for both stability conditions (see Figure 5d). The probability density of the vertical velocity, normalized hy the root-mean square velocity in the vertical, is displayed in Figure $5 \mathrm{~d}$ for both stability conditions. The flatness factors corresponding to these probability distributions are 3.15 (unstable) and 3.35 (stable), which are smaller than the wavelet flatness factors for production wavenumbers $(F F \sim 5)$. In order to investigate the influence of atmospheric stability on the spatial distribution of the velocity gradients, we consider the wavelet flatness factors for both stability conditions. For production wavenumbers $\left(2 \mathrm{~m}^{-1}-18 \mathrm{~m}^{-1}\right)$, the wavelet flatness factor is larger for unstable than for stable conditions (Figure $5 \mathrm{c}$ ). This indicates that the magnitudes of the gradients for unstable conditions are larger and appear more frequently in the vertical velocity signal than for stable conditions.

It is important to check whether the increased activity at smaller scales (i.e., increased $C V$ and $F F$ ) noted in Figures $5 c$ is a manifestation of the wavelet transform or a property of the turbulence signal. For that purpose, a comparison with a random Gaussian signal having the same probability distribution as experiment 1 was carried out. The details are presented and discussed in Appendix B. We remark (see Appendix B) that the increased activity at smaller scales is a property of the measured turbulence signals and not a manifestation of the orthonormal wavelet transform. 


\subsection{WAVELET STRUCTURE SKEWNESS}

The wavelet structure skewness is computed for various separation distances and scales using (35). We terminated the scale-axis at $10.88 \mathrm{~m}$ in order to have a statistically significant number of points (exceeding 512 points) for each averaging operation in (35). Also, these scales $(0.34 \mathrm{~m}-10.5 \mathrm{~m})$ correspond to the production scales in which the -1 power law was observed in the spectra of Figure 5a. Figure 2 is used to interpret the physical mechanism responsible for the sign of the wavelet structure skewness $(S S)$.

\subsubsection{Experiment 1: Unstable Conditions}

Figure 6a shows that buoyancy-generated turbulence is dominant in this case. This experiment is characterized by larger turbulent kinetic energy in the vertical than the other experiment (see Table I). The magnitude of the induced asymmetry (as characterized by $S S$ ) at the larger scales decays as larger and larger wavenumbers are approached. The decay of the buoyancy production asymmetry in the scale domain seems to be local and much more rapid when compared to Figure $6 \mathrm{~b}$.

\subsubsection{Experiment 2: Stable Case}

Experiment 2 was conducted under stable atmospheric conditions in which weak turbulent activity was noted. Unlike the unstable case, Figure 6-B indicates strong shear overturning with large asymmetry even for many scales that are smaller than $z^{-1}$. As expected, updraft-downdraft events are non-existent under stable conditions ( $S S$ is always negative) and the influence of the ground surface appears to affect scales as small as $z / 2$. This suggests that the anisotropy generated by shear production at the injection wavenumbers is still present, at least for the sampling frequency used in this experiment.

\section{Conclusions}

This study has focused on the spatial structure of the vertical velocity at production wavenumbers for unstable and stable stability conditions. It has been shown from dimensional arguments that the vertical velocity spectrum follows a -1 power law for wavenumbers comparable to production scales. Vertical velocity spectra measurements in the atmospheric surface layer confirmed the existence of the -1 power law for at least one decade. The use of orthonormal wavelets allowed decomposition of the measurements into space and scale. Contributions of each scale as well as the associated spatial variability were studied using dual spectra. It was found that a large spatial variation in the power exists and increases as the wavenumber increases. The spatial standard deviations in the power may be as large as three times the mean power within the -1 power-law spectral behavior.

The probability density functions of the vertical velocity for both stability conditions are found to be Gaussian. However, using the wavelet flatness factor, it 
Structure Skewness $280 / 1332$

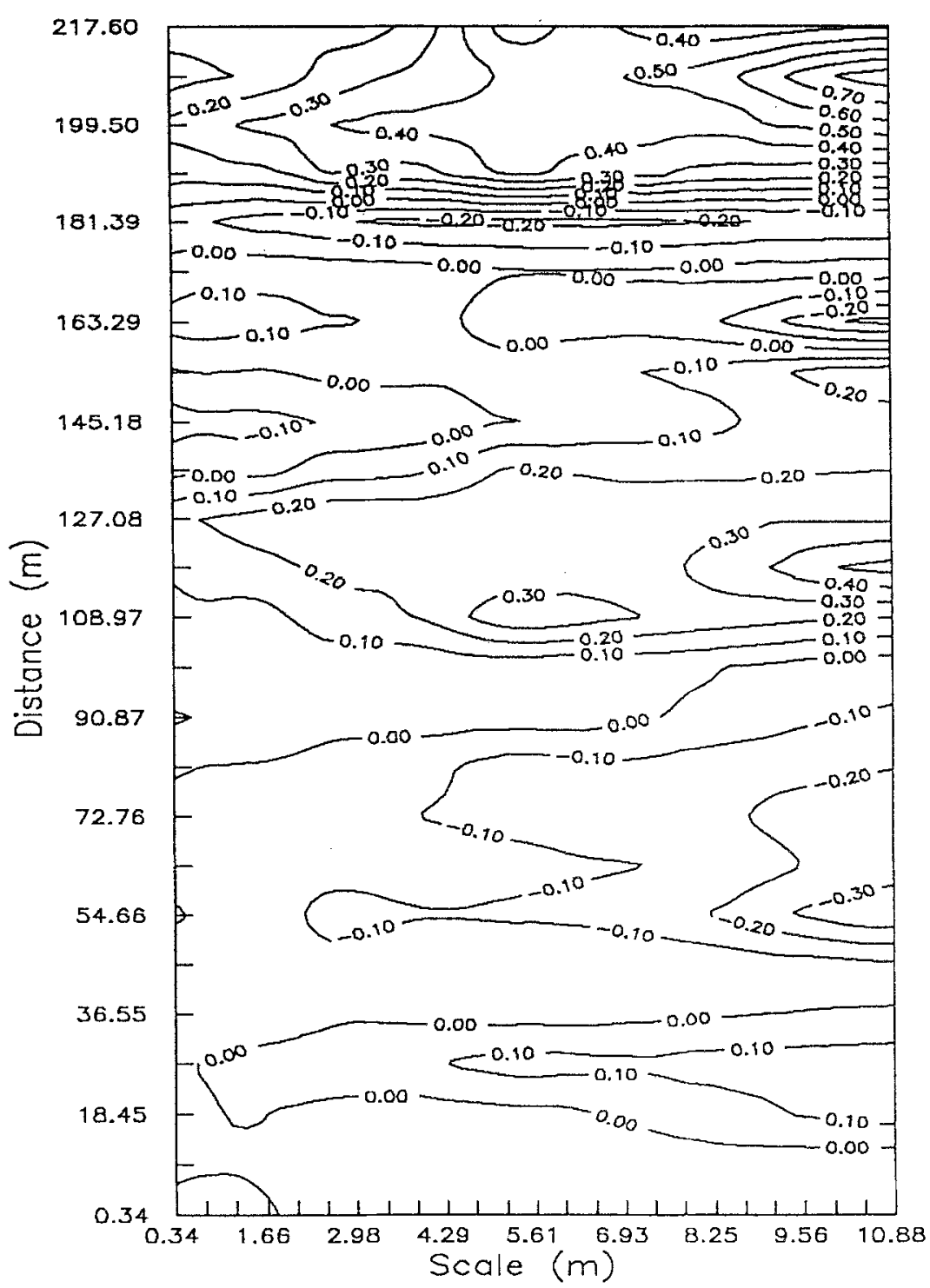

Fig. 6a. The wavelet structure skewness as a function of separation distance and scale for experiment 1 (unstable conditions). The contour plot was formed by simple linear interpolating. The production scales in which $\mathrm{a}-1$ power law occurs vary between 0.34 and $2.73 \mathrm{~m}$.

has been demonstrated that the horizontal gradients, at the production wavenumbers, are not Gaussian. The wavelet flatness factor generally increased, as the wavenumber increased indicating bigger and more frequently occuring large horizontal gradients at smaller scales (within the production spectrum). In order to check whether the increased turbulent activity at smaller scales (within the pro- 
Structure Skewness $285 / 2139$

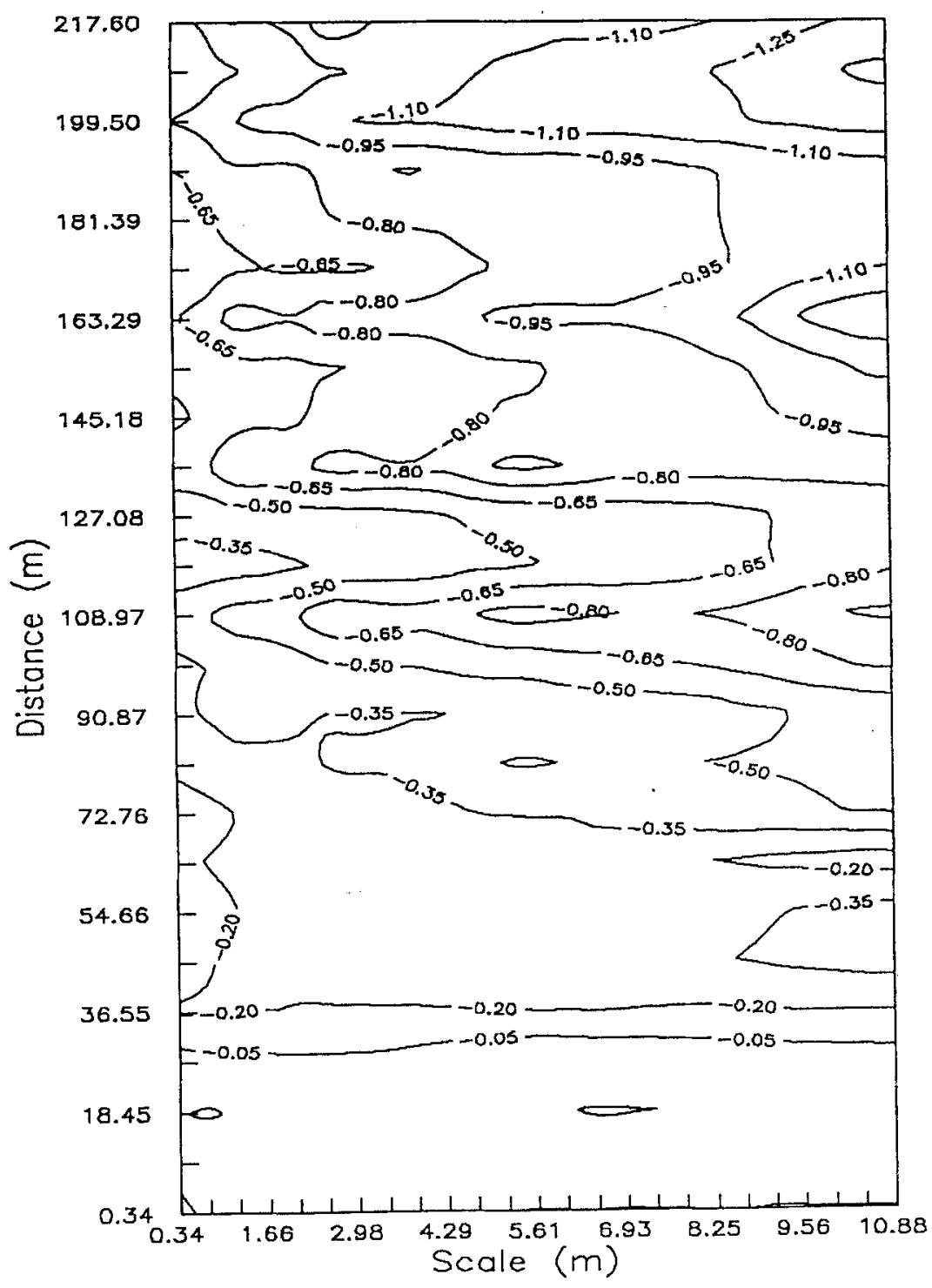

Fig. 6b. Same as Figure 6a but for experiment 2 (stable conditions).

duction spectrum) is due to the wavelet transform or a property of the measured vertical velocity, we performed a similar analysis on a synthetic signal. Hence, wavelet analysis was applied to a gaussian, random, $\delta$-correlated, synthetic signal with the same mean and variance as the measured turbulence signal. The results indicated that the flatness factor of the artificial signal is consistent with gaussian 
statistics at all wavenumbers. Therefore, what is revealed by the wavelet transform is a characteristic of turbulence and not of the transformation used.

A dimensionless structure skewness was used to identify the nature of the anisotropy within the-1 1 power-law production scales. It was noted that the nature of the anisotropy within the -1 power law is dependent on whether shear-overturning or updraft-downdraft events occur (and hence stability).

\section{Acknowledgments}

The authors gratefully acknowledge the help of Mike Mata in maintaining the field and discussions with John Albertson, Roger Shaw, Dennis Rolston and Larry Mahrt. This work was supported in part by the National Science Foundation (EAR93-04331), Kearney Foundation, California Water Resources Center, USGS and UC Davis superfund grant (5 P42ES04699-07).

\section{Appendix A: Fast Wavelet Transform for the Haar Basis}

In this Appendix, a simple and fast algorithm to compute the Haar wavelet coefficients is reviewed. More details are found in Beylkin et al. $(1991,1992)$.The Haar basis with $h_{j, k}(x)=2^{-j / 2} h\left(2^{-j} x-k\right)$ with $j, k \in Z$, is

$$
h(x)=\left[\begin{array}{ll}
1 & \text { for } 0 \leq x<1 / 2 \\
-1 & \text { for } 1 / 2 \leq x<1 \\
0 & \text { elsewhere }
\end{array}\right] .
$$

The wavelet coefficients $W^{(j+1)}(k)$ and the coarse-grained signal $S^{(j+1)}(k)$ at scale $j+1$ can be determined from the signal $S^{(j)}$ at scale $j$ using

$$
\begin{aligned}
& W^{(j+1)}(k)=\frac{1}{\sqrt{2}}\left[S^{(j)}(2 k-1)-S^{(j)}(2 k)\right], \\
& S^{(j+1)}(k)=\frac{1}{\sqrt{2}}\left[S^{(j)}(2 k-1)+S^{(j)}(2 k)\right],
\end{aligned}
$$

$k=0$ to $2^{M-j-1}-1$, and $M=\log _{2}(N)$, where $N$ is the number of samples. The above procedure, which is the basis for Fast Wavelet Transforms, requires about $N$ computations in comparison with the $N \log _{2} N$ computations for Fast Fourier Transforms (FFT).

\section{Appendix B: Comparison Between Turbulence and Gaussian Random Signals}

In this appendix, we apply the orthonormal wavelet transform to a random signal having the same mean and variance as the turbulence signal of experiment 1 , 


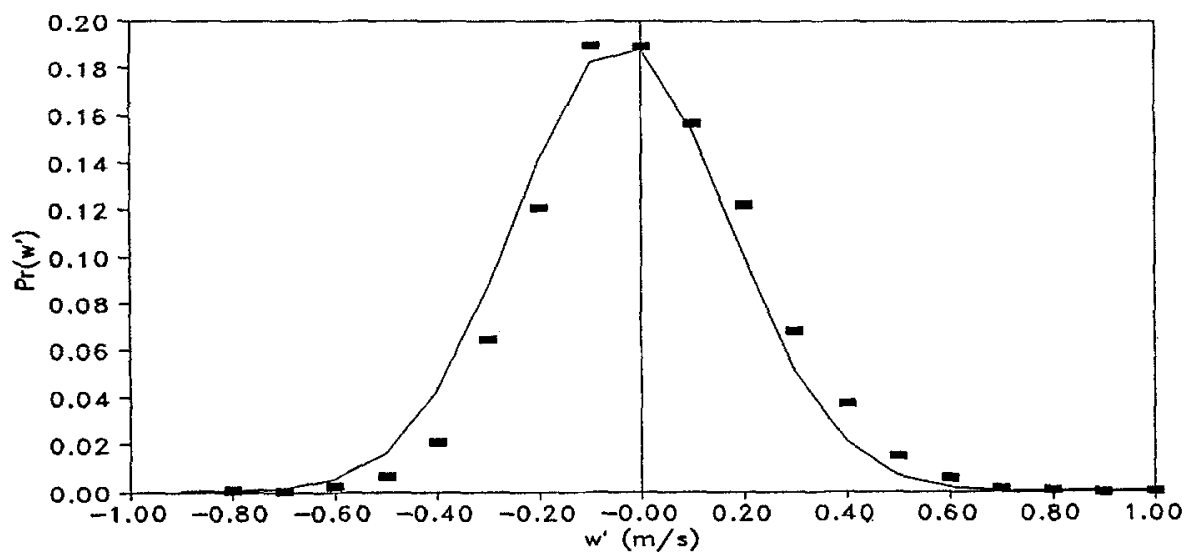

- Data Gaussion

Fig. 7a. A comparison between the frequency distribution of experiment 1 and a gaussian distribution with the same mean and variance.

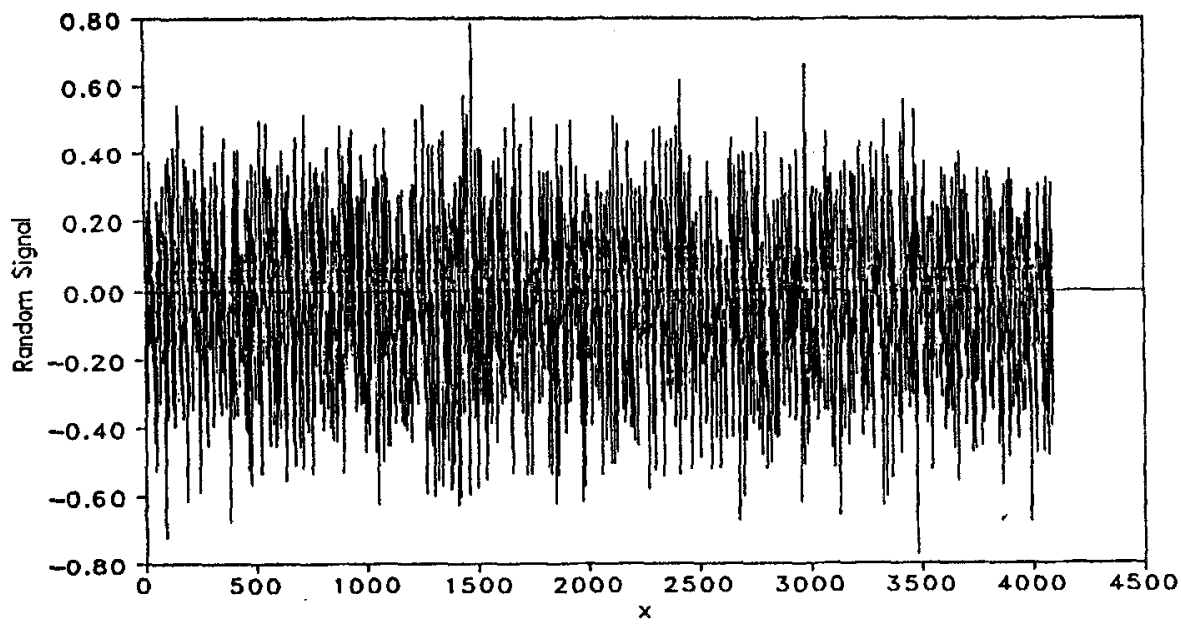

Fig. $7 \mathrm{~b}$. An artificial $\delta$-correlated random gaussian signal having the same mean and variance as the turbulence signal of experiment 1 .

which has a near gaussian behaviour. This comparison illustrates how the wavelet transform is capable of revealing some of the turbulence features in space and scale in relation to a $\delta$-correlated gaussian random process whose statistical and spectral properties are well known (e.g., Gelb, 1974, pp. 43; Lumley, 1970). In Figure 7a, the frequency distribution of $w^{\prime}$ (experiment 1 ) as well as a gaussian distribution fit for the same mean and variance are shown. Using the fitted distribution, a sequence of 4096 random numbers are generated; these are shown in Figure $7 \mathrm{~b}$ (see Press et al., 1990, pp. 203). The Multiresolution algorithm of Appendix A is then applied to this artificial signal and the wavelet spectrum as well as the flatness factor at 


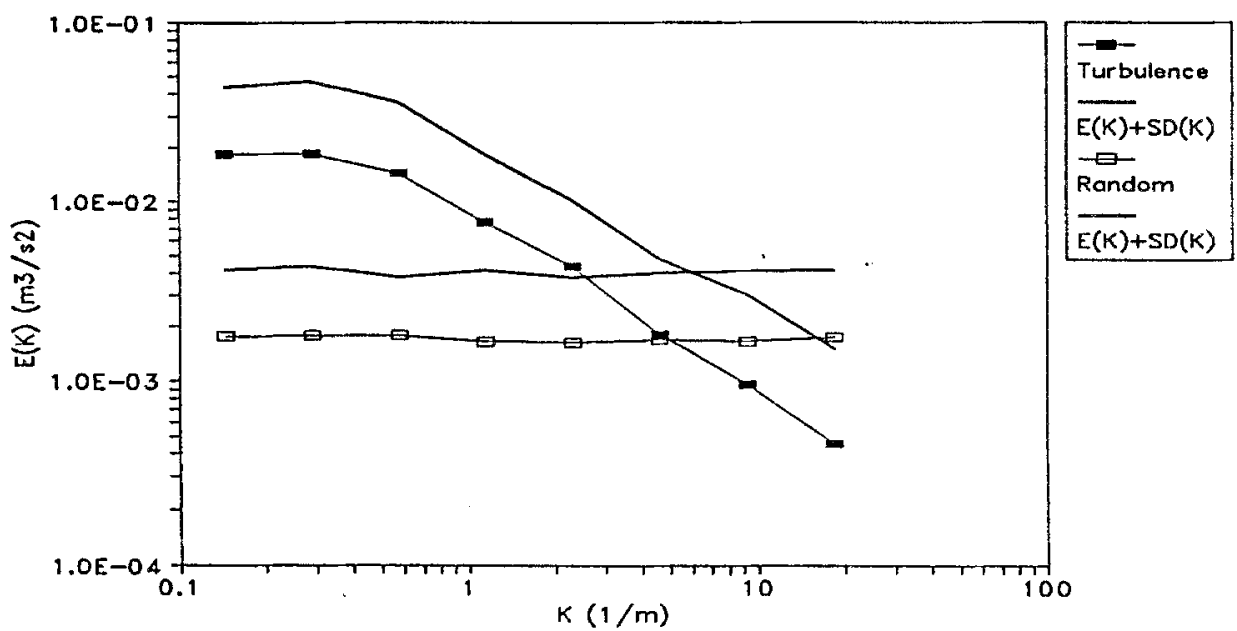

Fig. 7c. Comparison between the wavelet power spectra for the turbulence (experiment 1 ) and the artificial gaussian signal. Note that for the artificial signal, the power as well as the spatial standard deviation are constant.

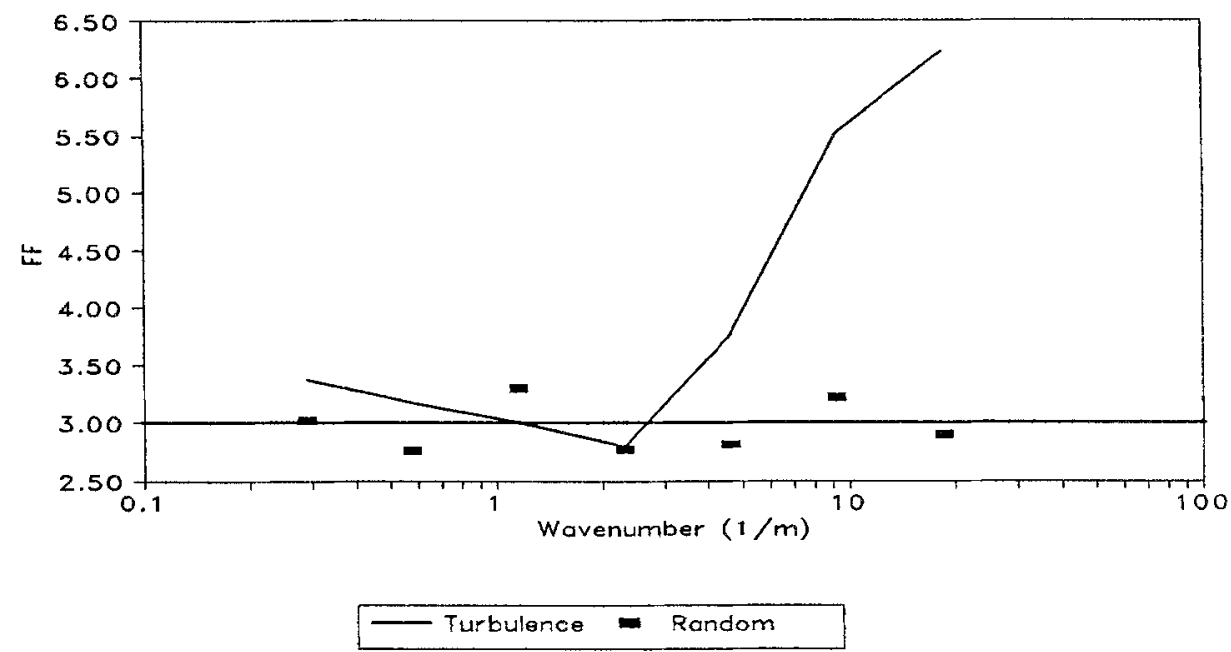

Fig. 7d. Comparison between the turbulence and the artificial signal spatial flatness factor $(F F)$. For the artificial signal, the flatness factor is a constant $\simeq 3$ for all wavenumbers.

various wavenumbers are computed. Figure $7 \mathrm{c}$ shows the dual spectrum of the random signal and the turbulence signal (experiment 1). Note that the power is uniform in the random signal as is typical of gaussian white noise processes. Also note that the standard deviation about the power is constant at every scale, unlike the turbulence signal which exhibits a distinct power-law behavior and variations in standard deviations that are wavenumber dependent. Figure $7 \mathrm{~d}$ shows the flatness factor at each scale for the artificial and the turbulence signal. For the artificial signal, the flatness factor is scattered around the line $F F=3$ at all wavenumbers, 
while the turbulence signal indicates a systematic increase in flatness factor as the wavenumber increases, indicating intensified spatial intermittency at higher wavenumbers (even though the probability distribution of $w^{\prime}$ for experiment 1 is nearly gaussian as noted in Figure 5d; see also She, 1991).

\section{References}

Argoul, F., Arneodo, A., Grasseau, G., Gagne, Y., Hopfinger, E. J., and Frisch, U.: 1989, 'Wavelet Analysis of Turbulence Reveals the Multifractal Nature of the Richardson Cascade', Nature 338, 51-53.

Antonia, R. A. and Raupach, R.: 1993, 'Spectral Scaling in a High Reynolds Number Laboratory Boundary Layer', Boundary-Layer Meteorol. 65, 289-306.

Barcy, E., Arneodo, A., Frisch, U., Gagne, G., and Hopfinger, E.: 1991, 'Wavelet Analysis of Fully Developed Turbulence Data and Measurement of Scaling Exponents', in O. Metais and M. Lesieur (eds.), Turbulence and Coherent Structures, Kluwer Academic Press, 450 pp.

Battle, G.: 1987, 'A Block Spin Construction of Ondelettes, Part I: Lemarie Functions', Comm. Math. Phys. 110, 601-615.

Battle, G.: 1992, 'Cardinal Spline Interpolation of the Block Spin Construction of Wavelets', in C. Chui (ed.), Wavelets: A Tutorial in Theory and Applications, Academic Press, Inc., 722 pp.

Betchov, R. and Yaglom, A. M.: 1971, 'Comments on the Theory of Similarity as Applied to Turbulence in an Unstably Stratified Fluid', Izv. Akad. Nauk. SSSR, Ser. Fiz. Atmosf. i Okeana, 7, 829-832, in English Translation of the Journal.

Beylkin, G., Coifman, R., and Rokhlin, V.: 1991, 'Fast Wavelet Transforms and Numerical Algorithms I', Comm. Pure and Appl. Math. XLIV, 141-183.

Beylkin, G., Coifman, R., and Rokhlin, V.: 1992, 'Wavelets in Numerical Analysis', in M. B. Ruskai, G. Beylkin, R. Coifman, I. Daubechies, S. Mallat, Y. Meyer, and L. Raphael (eds.), Wavelets and their Applications, Jones and Bartlett Publishers, Boston, 474 pp.

Brutsaert, W.: 1982, Evaporation into the Atmosphere: Theory, History and Applications, Kluwer Academic Puhlishers, $299 \mathrm{pp}$.

Busch, N. E.: 1973, 'The Surface Boundary Layer', Boundary-Layer Meteorol. 4, 213-240.

Chui, C. K.: 1992, An Introduction to Wavelets, Academic Press, Inc., 264 pp.

Collineau, S. and Brunet, Y.: 1993, 'Detection of Coherent Turbulent Motion above a Forested Canopy: Part I Wavelet Analysis', Boundary-Layer Meteorol. 65, 357-379.

Daubechies, I.: 1988, 'Orthonormal Bases of Compactly Supported Wavelets', Comm. Pure and Appl. Math. XLI, 909-996.

Daubechies, I.: Ten Lectures on Wavelets, 1992, CBMS-NSF Regional conference series in applied mathematics, S.I.A.M., 61, 357 pp.

David, G.: 1992, Wavelets and Singular Integrals on Curves and Surfaces, Springer-Verlag, 109 pp.

Erm, L. P. and Joubert, P. N.: 1991, 'Low-Reynolds-Number Turbulent Boundary Layer', J. Fluid Mech. 230, 1-44.

Everson, R., Sirovich, L., and Sreenivasan, K. R.: 1990, 'Wavelet Analysis of the Turbulent Jet', Physical Letters A 145, 314-322.

Farge, M.: 1992a, 'Wavelet Transforms and their Applications to Turbulence, Ann. Rev. Fluid Mech. $24,395-457$.

Farge, M.: 1992b, 'The Continuous Wavelet Transform of Two Dmensional Turbulent Flows', in M. B. Ruskai, G. Beylkin, R. Coifman, I. Daubechies, S. Mallat, Y. Meyer, and L. Raphael (eds.), Wavelets and their Applications, Jones and Bartlett Publishers, Boston, 474 pp.

Farge, M., Goirand, E., Meyer, Y., Pascal, F., and Wickerhauser, M. V.: 1992, 'Improved Predictability of Two Dimensional Turbulent Flows Using Wavelet Packet Compression', Fluid Dynamics Research, 10, 229-250.

Gelb, A., Kasper, J., Nash, R., Price, C., and Sutherland, A.: 1974, in A. Gelb (ed.), Applied Optimal Estimation, MTT Press, 374 pp. 
Grossmann, A., Kronland-Martinet, R., and Morlet, J.: 1989, 'Reading and Understanding Continuous Wavelet Transforms', in J. M. Combes, A. Grossmann, Ph. Tchamitchian (eds.), Wavelets: TimeFrequency Methods and Phase Space, Springer-Verlag, 315 pp.

Grossmann, A., and Morlet, J.: 1985, 'Decomposition of Functions into Wavelets of Constant Shape, and Related Transforms', in L. Streit (ed.), Mathematics + Physics, Lectures on Recent Results, World Scientific, Singapore.

Grossmann, A. and Morlet, J.: 1984, 'Decomposition of Hardy Functions into Square Integrable Wavelets of Constant Shape', SIAM J. Math. Anal., 15, 723-736.

Haar, A.: 1910, 'Zur theorie der orthogonalen funktionensysteme', Math. Ann. 69, 331-371.

Kader, B. A. and Yaglom, A. M.: 1984, 'Turbulent Structure of an Unstable Atmospheric Layer', in R. Z. Sagdeyev, Nonlinear and Turbulent Processes in Physics, Harwood Acad. Publ., 2, 829-845.

Kader, B. A. and Yaglom, A. M.: 1990, 'Mean Fields and Fluctuation Moments in Unstably Stratified Turbulent Boundary Layer', J. Fluid Mech. 212, 637-662.

Kader, B. A. and Yaglom, A. M.: 1991, 'Spectra and Correlation Functions of Surface Layer Atmospheric Turbulence in Unstahle Thermal Stratification', In O. Metais (ed.), Turbulence and Coherent Structures, Kluwer Academic Press, 450 pp.

Kaimal, J. C., Wyngaard, J. C., Izumi, Y., and Coté, O. R.: 1972, 'Spectral Characteristics of Surface Layer Turbulence', Quart. J. R. Meteorol. Soc. 98, 563-589.

Katul, G. G. and Parlange, M. B.: 1992, 'A Penman-Brutsaert Model for Wet Surface Evaporation', Water Resourc. Res. 28, 121-126.

Katul, G. G. and Parlange, M. B.: 1994, 'On the Active Role of Temperature in Surface Layer Turbulence', J. Atmos. Sci. 51 2181-2195.

Kolmogorov, A. N.: 1941, 'The Local Structure of Turbulence in Incompressible Viscous Fluid for Very Large Reynolds Numbers', Dokl. Akad. Nauk SSSR, 4, 299-303.

Korotkov, B. N.: 1976, 'Some Types of Local Self-Similarity of the Velocity Field of Wall Turbulent Flows', Izv. Akad. Nauk. SSSR, Ser. Mekh. Zhidk. i. Gaza, 6, 35-42.

Kraichnan, R.: 1991, 'Turbulent Cascade and Intermittency Growth', in J. Hunt, M. Phillips, and D. Williams (ed.), Turbulence and Stochastic Processes: Kolmogorov's Ideas 50 Years On, Roy. Soc., $240 \mathrm{pp}$.

Lemarie, P. G.: 1988, 'Ondelettes à Localisation Exponentielles', J. Math. Pure et Appl. 67, 227-236.

Lesieur, M.: 1987, Turbulence in Fluids, Martinus Nijhoff Publishers, 286 pp.

Liandrat, J. and Moret-Bailly, F.: 1990, 'The Wavelet Transform: Some Applications to Fluid Dynamics and Turbulence', Eur. J. Mech., B/Fluids, 9, 1-19.

Lumley, J.: 1965, 'Interpretation of Time Spectra Measured in High Intensity Shear Flows', Phys. Fluids 6, 1056-1062.

Lumley, J.: 1970, Stochastic Tools in Turhulence, Academic Press, 194 pp.

Madych, W. R.: 1992, 'Some Elementary Properties of Multiresolution Analysis of $L^{2}\left(R^{n}\right)$, in C. K. Chui (ed.), Wavelets: A Tutorial in Theory and Applications, Academic Press, Inc., 723 pp.

Mahrt, L. and Gamage, N.: 1987, 'Observations of Turbulence in Stratified Flow', J. Atmos. Sci. 44, $1106-1121$.

Mahrt, L.: 1991, 'Eddy Asymmetry in the Shear Heated Boundary Layer', J. Atmos. Sci. 48, 472-492.

Mallat, S.: 1989a, 'A Theory for Multiresolution Signal Decomposition: The Wavelet Representation', IEEE Trans. Pattern Analysis and Machine Intelligence, 11, 674-693.

Mallat, S.: 1989b, 'Multiresolution Approximations and Wavelet Orthonormal Bases of $L^{2}(R)$ ', Trans. Amer. Math, Soc. 315, 69-87.

Meneveau, C.: 1991a, 'Analysis of Turbulence in the Orthonormal Wavelet Representation', J. Fluid Mech. 232, 469-520.

Meneveau, C.: 1991b, 'Dual Spectra and Mixed Energy Cascade of Turbulence in the Wavelet Representation', Physical Review Letters 11, 1450-1453.

Metais, O. and Lesieur, M.: 1992, 'Spectral Large Eddy Simulation of Isotropic and Stably Stratified Turbulence', J. Fluid Mech. 239, 157-194.

Metais, O.: 1991, 'Large-Eddy Simulation of Turbulent Scalar: The Influence of Intermittency', in J. Jimenez (ed.), The Global Geometery of Turbulence, Plenum Press, pp. 155-166.

Meyer, Y.: 1989, 'Orthonormal Wavelets', in J. M. Combes, A. Grossmann, and Ph. Tchamitchian (eds.), Wavelets: Time-Frequency Methods and Phase Space, Springer-Verlag, $315 \mathrm{pp}$. 
Monin, A. S. and Obukhov, A. M.: 1954, 'Basic Laws of Turbulent Mixing in the Ground Layer of the Atmosphere', Tr. Geofiz. Inst. Akad. Nauk SSSR 151, 163-187.

Monin, A. S. and Yaglom, A. M.: 1971, in J. Lumley (ed.), Statistical Fluid Mechanics Vol. I, MIT Press, 768 pp.

Monin, A. S. and Yaglom, A. M.: 1975, in J. Lumley (ed.), Statistical Fluid Mechanics Vol. II, MTT Press, $874 \mathrm{pp}$.

Parlange, M. B. and Katul, G. G.: 1992, 'An Advection-Aridity Evaporation Model', Water Resour. Res. 28, 127-132.

Perry, A. E. and Abell, S. J.: 1975, 'Scaling Laws for Pipe Flow Turbulence', J. Fluid Mech. 67, 257-271.

Perry, A.E., Henbest, S., and Chong, M. S.: 1986, 'A Theoretical and Experimental Study of Wall Turbulence', J. Fluid Mech. 165, 163-199.

Powell, D. and Elderkin, C. E.: 1974, 'An Investigation of the Application of Taylor's Hypothesis to Atmospheric Boundary Layer Turbulence', J. Atmos. Sci. 31, 990-1002.

Press, W. H., Flannery, B. P., Teukolsky, S. A., and Vetterling, W. T.: 1990, Numerical Receipes: The Art of Scientific Computing, Cambridge University Press, 702 pp.

Raupach, M. R., Antonia, R. A., and Rajagopalan, S.: 1991, 'Rough-Wall Turbulent Boundary Layers', Appl.Mech. Rev. 44, 1-25.

She, Z.: 1991, 'Intermittency and Non-Gaussian Statistics in Turbulence', Fluid Dynamics Research $8,143-158$.

Sirivat, A. and Warhaft, Z.: 1983, 'The Effect of a Passive Cross-Stream Temperature Gradient on the Evolution of Temperature Variance and Heat Flux in Grid Turbulence', J. Fluid Mech. 128, 323-346.

Stanisic M. M.: 1985, The Mathematical Theory of Turbulence, Springer-Verlag, $429 \mathrm{pp}$.

Stull, R.: 1988, An Introduction to Boundary Layer Meteorology, Kluwer Academic Publishers, 666 pp.

Taylor, G. I.: 1938, 'The Spectrum of Turbulence', Proc. Roy. Soc., A CLXIV, 476-490.

Tchen, C. M.: 1953, 'On the Spectrum of Energy in Turbulent Shear Flow', J. Res. National Bureau of Standards 50, 51-62.

Tennekes, H. and Lumley, J.: 1972, A First Course in Turbulence, MIT Press, 300 pp.

Yamada, M. and Ohkitani, K.: 1990, 'Orthonormal Expansion and its Application to Turbulence', Prog. Theor. Phys.: Progress Letters 86, 819-823.

Yamada, M. and Ohkitani, K.: 1991a, 'Orthonormal Wavelet Analysis of Turbulence', Fluid Dynamics Research 8, 101-115.

Yamada, M. and Ohkitani, K.: 1991b, 'An Identification of Energy Cascade in Turbulence by Orthonormal Wavelet Analysis', Prog. Theor. Phys. 86, 799-815.

Zilitinkevich, S. S.: 1971, 'On the Turbulence and Diffusion Under Free Convection Conditions', Izv. Akad. Nauk. SSSR, Ser. Fiz. Atmosf. i Okeana, 7, 1263-1269. 